\title{
The impact a first episode of major depression has on marital dissatisfaction: Is remission associated with improvement in dissatisfaction?
}

\author{
Jaime Moyá, MD Ph.D.* \\ Adrián Cano Prous, MD Ph.D.** \\ Antonio Seva Fernández, MD Ph.D.*** \\ Álvaro Alonso, MD Ph.D.**** \\ * Child and Adolescent Psychiatry \\ and Psychology Department, Hospital Clinic, \\ Barcelona \\ ** Department of Psychiatry, University \\ of Navarra Clinic \\ *** Department of Medicine, Psychiatry and \\ Dermatology, University of Zaragoza \\ **** Division of Epidemiology and \\ Community Health, School of Public Health, \\ University of Minnesota
}

SPAIN

USA

\footnotetext{
ABSTRACT - Background and Objectives: Prior research suggests that marital dissatisfaction is associated with Major Depression (MD). The purpose of this study was to investigate whether remission from a first episode of MD is associated with improvement in marital dissatisfaction and whether the degree of marital dissatisfaction at the time of diagnosis has an influence on the outcome of MD.

Methods: The Hamilton Rating Scale for Depression, the Dyadic Adjustment Scale and the Areas of Change Questionnaire were administered to 59 married couples in which one member fulfilled DSM-IV criteria for a first Episode of MD, but her husband (or his wife) did not suffer any mental disorder, and to 53 control couples at 6 outpatient clinics, at baseline and after a 6-months follow-up.

Results: The level of marital dissatisfaction in couples with a persistent depressed participant decreased in comparison to couples without a depressed subject ( $p<0.05 \mathrm{CI}$ : 10.6 and -0.2 ), but there were no significant differences between couples in which the depressed spouse recovered from MD and comparison couples. Regardless of MD's severity, high levels of satisfaction were associated with a higher probability to recover.

Limitations: The follow-up period was short and we assessed the level of marital dissatisfaction using self-report measures.
} 
Conclusion: This study shows that MD has an influence on Marital Satisfaction even at a very early stage of the Depressive Disorder. What is more, changes in any of the two factors (MD or marital dissatisfaction) influenced the other factor's course; thus an effective intervention on MD may have a positive impact on marital dissatisfaction as well.

Received: 26 January 2009

Revised: 14 September 2009

Accepted: 28 September 2009

\section{Introduction}

Research demonstrates that marital dissatisfaction is strongly associated with diagnostic Depression ${ }^{1}$. However, most studies evaluating this topic have been cross-sectional $^{2-9}$ and have not addressed the temporal nature of this association. In fact, only few longitudinal studies have been published ${ }^{10-13}$ and some issues remain still unresolved or controversial.

First, most existing studies have evaluated the association between marital dissatisfaction and depressive symptoms, not between marital dissatisfaction and Major Depression $(M D)$, which might have different characteristics. According to Whisman's review ${ }^{14}$, findings have been mixed and inconclusive, but there is some evidence that marital dissatisfaction may be a good predictor of Depression. Nonetheless, most existing studies recruited both participants experiencing a recurrent episode of depression and participants experiencing a first episode. But, previous episodes appear to affect the risk of recurrence, shape the trajectory of early adulthood transitions ${ }^{15}$ and even contribute directly and indirectly to marital dissatisfaction. The question arises whether a single episode can affect marital functioning or whether the effect of MD on marital functioning is rather present at a later stage of the Depressive Disorder (e.g. when subjects ex- perience recurrences) and takes place after a larger period of enduring depressive symptoms. Consequently, it might be interesting to assess only participants with first episodes and examine the effect these episodes have on marital relationships.

Up to date, there has been a long-standing controversy as to which factor precedes, or even precipitates, the other one. On the one hand, Beach et al. ${ }^{16}$ found marital distress can predict Depression. By contrast, UlrichJakubowski et al. ${ }^{17}$ argued that Depression leads to marital distress. Therefore, it is feasible that there may be two possible orders of occurrence. Under certain circumstances Depression is likely to lead to marital dissatisfaction, but on other occasions marital dissatisfaction may precede Depression. Given that the chronology of this association is probably variable it might be more important to examine if there is a reciprocal influence between Depression and Marital Dissatisfaction once both factors coexist rather than studying the issue of which comes first. Unfortunately, there has been little attention directed towards this matter which can be of great interest since the improvement of one factor (e.g. after therapeutic intervention) could have also an impact on the other one.

Finally, the great majority of research on this topic has been carried out in the United Stated of America. Yet, culture and social environment might play an important role ${ }^{18}$. For 
that reason, a step toward improving the understanding of a topic which probably exists world-wide is to evaluate couples in Spain.

Taking the existing evidence into account, the present study attempts to address the following issues:

1. Investigate whether remission from a First Episode of MD was associated with improvement in dissatisfaction.

2. Explore whether the degree of marital dissatisfaction at the time of diagnosis has an influence on the outcome of the First Episode of MD.

Our hypotheses are that:

- A positive outcome of the First Episode of MD is associated with an improvement in marital satisfaction.

- Depressed subjects in a couple with lower levels of marital dissatisfaction at onset of a First Episode of MD are more likely to recover from the Episode.

\section{Methods}

\section{Inclusion/ Exclusion Criteria}

Participants were eligible for inclusion if (1) aged between 18 and 65 years; (2) married for at least two years; (3) had given informed consent; (4) one spouse met DSM-IV criteria for First Episode of MD when first assessed, but her husband (or his wife) failed to meet those criteria; (5) the depressed participant scored $>14$ and his/her spouse $<8$ on the Hamilton Rating Scale for Depression.

Couples were excluded if prior to the initial assessment any participant (1) had been diagnosed with any severe mental disorder; (2) had significant medical conditions; (3) was pregnant; (4) was admitted to hospital.

\section{Participants}

Participants in this study were evaluated twice, at the time they were first assessed by a mental health professional and diagnosed with the first episode of MD and six months later. All participants were drawn from 154 subjects who attended 6 different clinics: the Psychiatry Outpatient Department at the University Clinic of Navarre, the Casco Viejo Mental Health Clinic, the Ermitagaña Mental Health Clinic, the Tudela Mental Health Clinic, the Azagra Health Service and a private Mental Health clinic in Barcelona. Most subjects were referred by their family care physician, but some were self-referred.

Among those initial 154 couples, 61 (40\%) did not meet our inclusion criteria and 22 (14\%) refused to participate. Six months later 12 couples did not complete the second assessment ( 5 refused to continue in the study and 7 were excluded). As a result, 59 couples of the initial 71 couples $(83 \%)$ were reinterviewed (see flow chart 1).

Wives $(62 \%)$ were more frequently the ones who were depressed (vs 38\% husbands).

There were no significant differences in sociodemographic or marital functioning data between either the remaining control couples or the remaining couples with a depressed spouse and dropouts (data not shown but available on request).

The final sample consisted of non-minority Spanish couples (95\% Caucasian). Participants had a mean age of 44 years $(\mathrm{SE}=$ 1.3), a mean duration of their marriage of 17 years (CI 95\%: 20.1-14.3) and an average of 2-3 children (54.9\%), 76\% were living only with their children at home (no other relatives at home) and a great majority of them were employed $(90.1 \%)$.

During follow-up, $93 \%$ of depressed subjects were treated with antidepressants (almost 


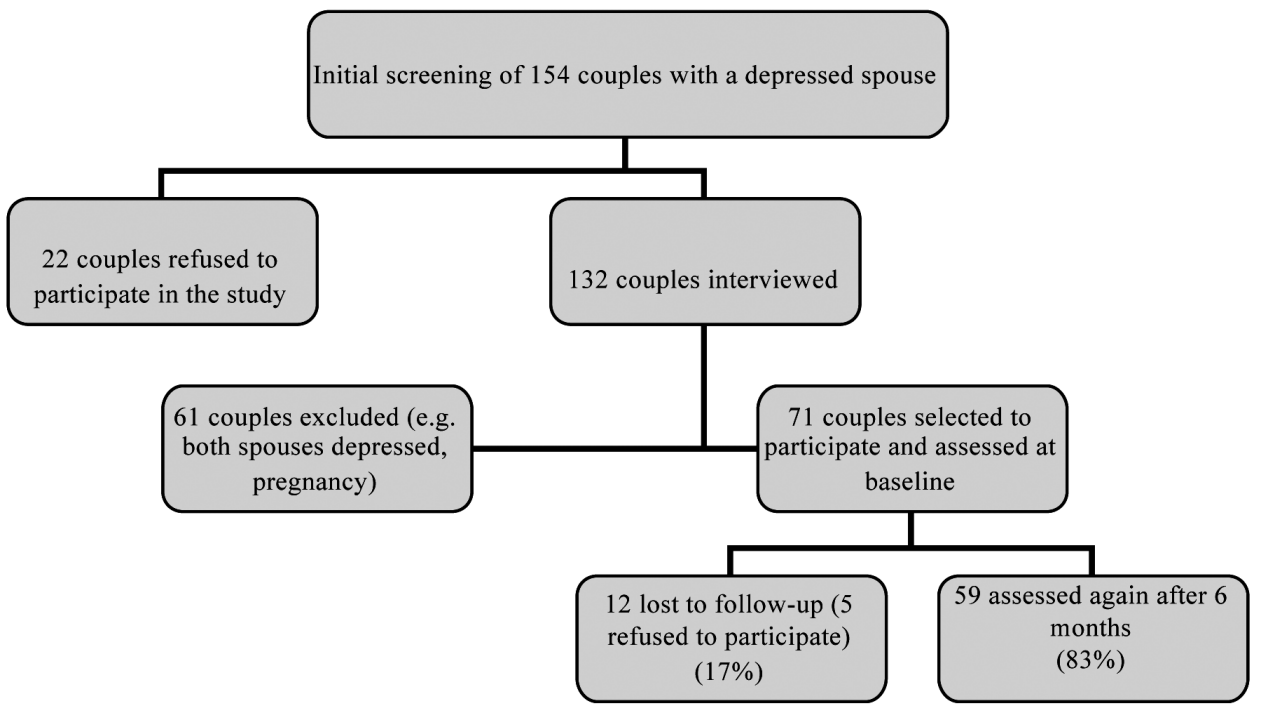

Flow Chart 1. Couples with a depressed spouse

exclusively SSRI) and 29\% were treated with cognitive-behavioural psychotherapy as well. Thus, 36 depressed spouses remitted $(61 \%)$ and the mean score of the Hamilton Rating Scale for Depression 17 item varied from 19.8 $(\mathrm{SE}=0.5)$ to $8.9(\mathrm{SE}=0.9)(\mathrm{p}<0.001)$ and the GAF score from $67.9(\mathrm{SE}=0.8)$ to 78.3 $(\mathrm{SE}=1.1)$ between both assessments.

\section{Comparison couples}

Signs about the study were posted at the University Hospital and members of staff and visitors were informed about the project. 78 control couples were initially recruited but $8(12 \%)$ refused to participate. 70 were assessed after the initial screening and 60 selected to participate and mainly home-interviewed of which $53(88 \%)$ provided data again six months later (see flow chart 2).

Every depressed subject was matched with a non-depressed spouse of the control couples for age $(\mathrm{M}=44.8$ years, $\mathrm{SE}=1.2$ versus $\mathrm{M}=42.1$ years, $\mathrm{SE}=1.5$ respectively) and gender. Overall, the two groups of couples did not differ on age, duration of marriage and number of children. However, control couples had more years of education $(91 \%$ DC university degree vs 56\% depressed couples) (table 1).

\section{Procedure}

All subjects referred to each one of the six recruitment centres of this project were routinely assessed and diagnosed by an experienced psychiatrist. Those who were eligible for this investigation were invited to take part in a longitudinal study of depression and marriage. Then, and if both spouses gave permission, they were referred to another experienced psychiatrist, the first author of this paper (JM), who assessed them again within the next 7 days.

All recruited couples were interviewed by the same psychiatrist (JM) both at baseline and six months later and spouses were 


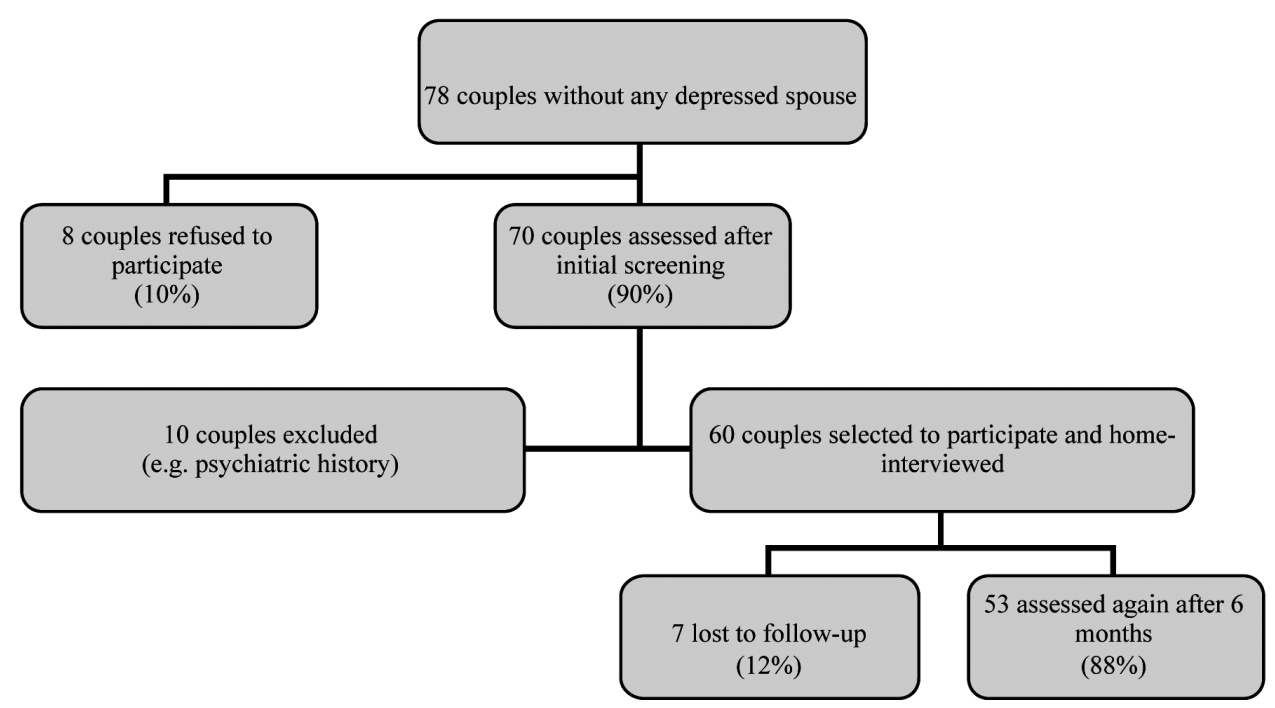

Flow Chart 2. Control couples

Table 1

Characteristics of the study sample

\begin{tabular}{lccc} 
& $\begin{array}{c}\text { Depressed couples } \\
(\mathrm{n}=71)\end{array}$ & $\begin{array}{c}\text { Control couples } \\
(\mathrm{n}=60)\end{array}$ & $\begin{array}{c}\text { Bivariate } \\
\text { Comparison }\end{array}$ \\
\hline Age, mean years \pm SD & $39.5 \pm 11.9$ & $42.6 \pm 11.8$ & $40.2 \pm 12.1$ \\
\hline Race or ethnic group & $\begin{array}{c}\text { 95\% Caucasian } \\
5 \% \text { others }\end{array}$ & $\begin{array}{c}94 \% \text { Caucasian } \\
6 \% \text { others }\end{array}$ & 0.67 \\
\hline Mean age & $44.8(1.2)$ & $42.1(1.5)$ & 0.16 \\
\hline Duration of marriage (in years) & $17.2(1.4)$ & $16.0(1.6)$ & 0.58 \\
\hline Number of children & & & \\
0 & $12(16.9 \%)$ & $17(28.3 \%)$ & 0.37 \\
$1-2$ & $39(54.9 \%)$ & $28(46.7 \%)$ & \\
$3-4$ & $15(21.1 \%)$ & $9(15.0 \%)$ & \\
$>4$ & $5(7 \%)$ & $6(10.0 \%)$ & \\
\hline Education & & $2(3.3 \%)$ & \\
Primary education (up to age 16 years) & $10(14.1 \%)$ & $3(5.0 \%)$ & \\
Secundary (up to age 18-20 years) & $21(29.6 \%)$ & $55(91.7 \%)$ & 0.13 \\
Higher education (university) & $40(56.3 \%)$ & & \\
\hline Living with: & & $42(70.0 \%)$ & $1(1.7 \%)$ \\
- Children & $54(76.1 \%)$ & $17(28.3 \%)$ & \\
- Children \& grandparent & $5(7.0 \%)$ & $12(16.9 \%)$ & \\
- Couple only & & & \\
\hline
\end{tabular}

$\mathrm{t}$ Student was used to compare age and years of marriage. U Mann Whitney was chosen to compare education, people living at home and number of children. Race and ethnic group was compared using Chi-square. 
always assessed separately. It was confirmed that both spouses met the inclusion criteria during the initial appointment and the purpose and aims of the study were explained. Additionally, spouses were given a telephone number for any enquiries they had in relation to the study or their disorder. Six months later, all couples were contacted again, by telephone or by post, and were assessed at the same clinical setting where the first assessment took place. However, some couples could not go to the clinics and were home-interviewed.

Study procedures were done with full approval of the Research Board and the Ethical Committee of the University of Navarre. Participants were always guided through full informed-consent procedures and did not get any compensation for their collaboration.

Whenever possible, questionnaires and scales which had been previously used in Spain were administered (Structured Clinical Interview for DSM-IV ${ }^{19}$, Hamilton Rating Scale for Depression ${ }^{20}$, Global Assessment of Functioning Scale ${ }^{21}$ ). Marital satisfaction measures were translated and back-translated from the original.

During the second assessment, remission was defined as the outcome when DSM-IV criteria for First Episode of MD were not fulfilled, the score on the Global Assessment of Function (GAF) $\geq 80$ and the score on the Hamilton Rating Scale for Depression $\leq 8$. Any clinical presentation different from this was considered non-remission.

\section{Measures}

\section{Diagnostic Interviews}

The Structured Clinical Interview for DSMIV Axis I psychiatric diagnoses ${ }^{19}$ was chosen for its thoroughness in diagnostic assessment and the part referring to MD was administered by a trained mental health professional (JM). It has been found to be a valid scale 22 and has been translated into Spanish ${ }^{23}$.

\section{Depressive symptoms}

The Hamilton Rating Scale for Depres$\operatorname{sion}^{20}$ is a multiple choice questionnaire which rates the severity of MD. It has been shown to be a reliable interviewer-administered instrument ${ }^{24}$, widely used and validated into Spanish 25 .

\section{Global Functioning}

The Global Assessment of Functioning Scale $(G A F)^{21}$ is presented and described in the DSM-IV. This instrument has been found to be rated reliably after minimal training ${ }^{26}$ and it was chosen used to assess participant's impairment.

\section{Marital satisfaction}

Dyadic Adjustment Scale $(D A S)^{27}$. This self-report 32-item questionnaire provides an assessment of perceived marital quality and includes four subscales: Dyadic Satisfaction, Dyadic Cohesion, Dyadic Consensus, and Affectional Expression and one global score. Higher scores indicate greater satisfaction. Crohnbach's alpha and the total scale's testretest reliability after 11 weeks have been found to be $0.96^{27}$. This scale has also shown to be adequate to differentiate distressed couples from those who are well adjusted ${ }^{28}$, can be answered in 10 minutes and is highly correlated to its predecessor the Marital Adjustment Test given that almost $70 \%$ of its items are included in the $\mathrm{DAS}^{29}$. 
Areas of Change Questionnaire (ACQ). This inventory consists of 68 items $^{30}$ and measures (a) the extent to which one desires his/her spouse to change in several problem areas and (b) the judgment as to the degree the other spouse might desire one to change in those areas. Test-retest reliability has been found to be 0.96 after 6 weeks, the internal consistency of $0.89^{31}$ and to be negatively correlated with the Marital Adjustment Test $^{32}$. A particular strength of the scale is showing both the negative and positive areas in couple relationships ${ }^{33}$.

\section{Statistical analysis}

All measures were analyzed using the Statistical Package for the Social Sciences (SPSS version 14; SPSS Inc, Chicago, Illinois). Baseline characteristics of all participants were compared between the treatment groups using $t$ tests or chi-square tests as appropriate for the data. In all analyses, results with P0.05 for a 2-tailed interpretation were considered statistically significant. The main outcome variables were DAS and ACQ scores at the second visit, and remission of the depressive episode at the second visit. We used multiple lineal regressions to study the influence of Major Depression on marital satisfaction (DAS and ACQ), with control couples as the reference group. The statistical significance of remission on marital functioning variables was tested with logistic regression analysis and odds ratios (ORs) and 95\% confidence intervals (CIs) were estimated, with remission/not remission as the outcome variable, and levels of DAS and ACQ at the initial visit as main predictors. The linear association ( $p$ for trend) between marital satisfaction and depression remission was tested including DAS or ACQ scores in the models as continuous variables.
The statistical measure chosen to describe the dispersion of values was the Mean Standard Error.

\section{Results}

The statistical difference between the depressed subject's marital satisfaction (Mean $=106.8, \mathrm{SE}=2.8)$ and his/ her spouse's $(\mathrm{M}=$ 107.7, SE = 2.3) was not significant ( $(70)$, $\mathrm{p}>0.05)$. In addition, all global score and subscales were between the $45-50^{\text {th }}$ percentile of the standardized distribution. Since both subjects within a "depressed couple" experienced similar levels of satisfaction, we calculated a mean for each couple and used this value for further analyses. The analysis unit of our study was the couple.

Overall, depressed couples' marital adjustment scores hardly changed during the study period (at baseline 107.7 vs 107.3 at follow-up) but they were significantly less satisfied than control couples (at baseline 120.5 vs 119.5 at follow-up) throughout the study ( $\mathrm{t}(70), \mathrm{p}>0.05)$. What is more, those couples in which Depression remitted were significantly more satisfied than those with persistent MD (111.8 vs 101.3; $\mathrm{t}(70), \mathrm{p}>0.05$ ). However, the level of satisfaction couples with remission reported did not reach the one controls had (111.8 vs 119.5).

\section{The influence of the Depressive Episode on marital satisfaction}

In order to test our first hypothesis, we divided the sample in three groups: control couples, couples with a remitted depressed subject and couples in which the depressed subject didn't remit and performed a multiple lineal regression to test. We used two 
"dummy variables", the independent variable was the group couples belonged to and the main dependent variable was the difference in marital satisfaction.

The level of marital satisfaction in couples with a persistent depressed participant decreased in comparison to control couples, controlling for marital satisfaction during the first assessment and the mean age of the couple. Our results also showed that marital satisfaction did not significantly decrease for the control couples. No significant differences between remitted and control couples were found. (see table 2).

Consistent with the previous analysis, ACQ questionnaire scores show that couples with a non-remitting depressed subject have a tendency to wish more changes in their relationship compared to the other two groups. Yet, this difference is not statistically significant $(\mathrm{p}=0.10$ : CI -0.6 and +7.2$)$ (table 3$)$.

\section{The influence of marital quality on the outcome of the depressive episode}

Further, we conducted three different logistic regression analyses considering remission of the First Episode of MD as the outcome and the degree of marital satisfaction during the first assessment, measured by the DAS and ACQ questionnaires, as the main independent variable.

First, we examined if marital satisfaction level perceived by both spouses was related to outcome. Using couple's global DAS score or their ACQ scores, we found the degree of marital satisfaction had an influence on the likelihood of remission, regardless of the severity of the First Episode of MD. High scores on marital satisfaction were associated with a higher probability to recover and, in fact, above 117 points, the odds ratio was 5.8, adjusted for age and gender ( $\mathrm{p}$ of trend $\leq 0.05$ ). ( see table 4 .).

Table 2

Association between couple characteristics and marital satisfaction. Coefficient corresponds to differences in DAS score at the second assessment

\begin{tabular}{lcccc} 
& Coefficient & CI 95\% & $\mathrm{p}^{*}$ \\
\hline Couples without a depressed subject & (reference) & & \\
\hline Couples with a remitted depressed subject & +2.3 & -1.9 and +6.6 & 0.28 \\
\hline Couples with a persistent depressed subject & -5.4 & -10.6 and -0.2 & 0.04
\end{tabular}

$\mathrm{p}^{*}=\mathrm{p}$-value, significance of the test, multiple regression analysis, adjusted for DAS during the first assessment and average age, CI: confidence interval.

Table 3

Association between couple characteristics and problem areas. Coefficient correspond to differences in ACQ score at the second assessment

\begin{tabular}{lcccc} 
& Coefficient & CI 95\% & $\mathrm{p}^{*}$ \\
\hline Couples without a depressed subject & (reference) & & \\
\hline Couples with a responding depressed subject & -0.3 & -3.3 and +2.8 & 0.87 \\
\hline Couples with a non-responding depressed subject & +3.3 & -0.6 and +7.2 & 0.10
\end{tabular}

$\mathrm{p}^{*}=\mathrm{p}$-value, significance of the test, multiple regression analysis, adjusted for ACQ during the first assessment and age media, CI: confidence interval. 


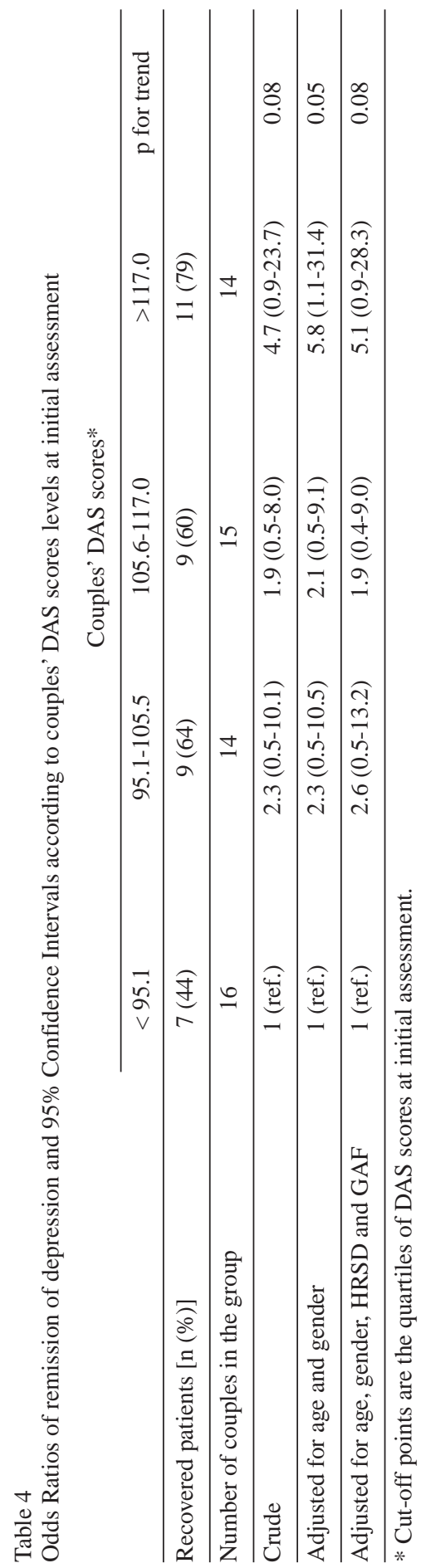

Consistent with the previous analysis, we found that wishing more changes in potentially problematic areas was associated to a lower likelihood to recover, adjusted for age and gender ( $\mathrm{p}$ of trend $\leq 0.05$ ) (see table 5).

Finally, we also tried to determine the degree to which the depressed spouse's view of his/ her marital relationship was related to the outcome. Another logistic regression was performed and the level of satisfaction predicted the outcome (for depressed DAS' score $>119 \mathrm{p}$ for trend 0.03 ). This seems to represent the fact that based on the odds ratio depressed patients with high levels of marital satisfaction at the time of diagnosis were 20.5 more likely to recover from depression (table 6).

\section{Discussion}

Consistent with our hypothesis, the present study provides evidence that there is an improvement in marital dissatisfaction associated with remission from Major Depression six months after a patient is diagnosed with a first episode.

We also found that lack of remission was associated with continuing marital dissatisfaction. Consequently, changes in any of the two factors influenced the other factor's course. Nonetheless, depressed couples' satisfaction levels did not reach the level control couples had. It is obvious that these results depend, to some extent, on the choice of an adequate control group. We chose a non-psychiatric group, a strategy already used by Stravynsky et al. ${ }^{34}$, and the baseline level of control couples was similar to the one previously reported by Vega et al. ${ }^{35}$ in Spain. Thus, our findings confirm that the association exists in a Southern European culture 

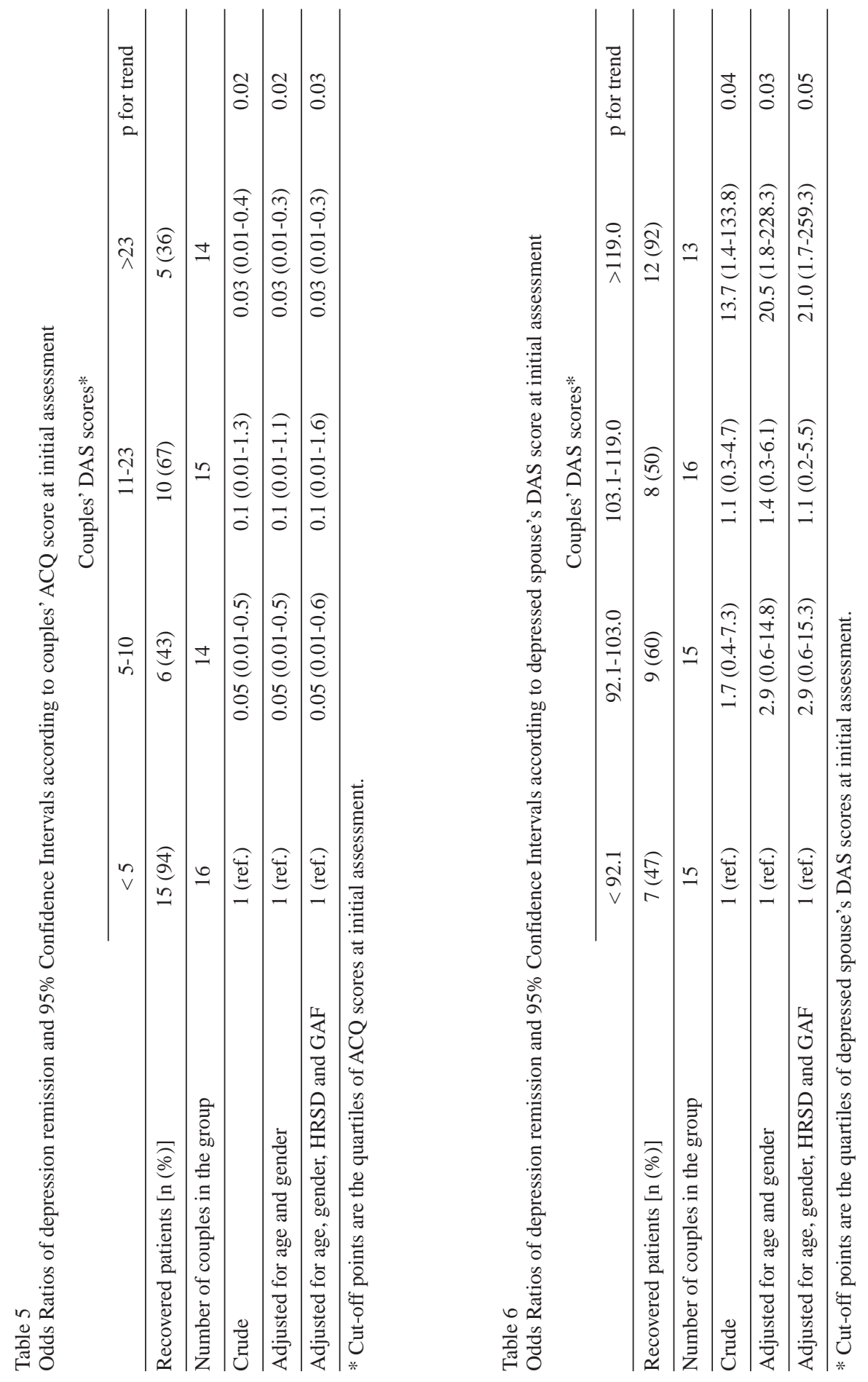
which is likely to be, to some extent, different to the Anglo Saxon culture. However, more research is needed to examine cultural differences which could influence the outcome and characteristics of this association.

Our findings are also in agreement with Coyne's and Benazon's ${ }^{15}$ view that marital dissatisfaction might affect the resolution of Depression and Hooley and Teasdale's ${ }^{36}$ results which demonstrated that not only marital distress, but also expressed emotion could predict the clinical outcome of depressed participants. However, unlike them, we did not recruit individuals at Inpatient Units. Indeed, it might be essential not to isolate the depressed individual from his/her family if we are going to study the interaction with his/her partner.

Moreover, taking into account the symptomatic course of MD and the fluctuations of marital satisfaction, longitudinal studies are more appropriate to understand the association. This study's goal was to determine if remission from MD was associated with improvement in dissatisfaction and to analyze if both factors were influencing each other, rather than examining the issue of causality.

It is doubtful, but possible, that the time participants in our study were already having depressive symptoms and waiting to be assessed by a psychiatrist was enough to influence their marital satisfaction. Conversely, couples who had a depressed spouse at the baseline assessment may have had lower levels of satisfaction some time before the onset of Depression, though most individuals did not mention marital dissatisfaction as one of their main concerns during the initial assessment.
All in all, what may be important, on the practical level, is to know if an intervention on one factor can improve the other condition at a very early stage. As suggested by Whisman ${ }^{14}$, future efforts should also try to find the mediators and moderators of this association. It is plausible that those factors might be possible areas for therapeutic intervention. In addition, there is a need to examine individuals before the onset of a Depressive Disorder and to determine if there are characteristics in their relationship which are related to the onset of MD.

\section{Limitations}

The number of couples in our sample is modest; power to detect differences is limited. In addition, couples were not asked to do laboratory interaction tasks and the self-report measures we used might not cope with the social desirability bias. Finally, marital satisfaction instruments were translated into Spanish for this study.

\section{Funding source}

Funding for this study was provided by two grants: University of Navarra Institute of Family Sciences Grant and Navarra Institute of Science and Technology Grant. Those institutes had no further role in this project's design, collection, analysis or interpretation of data, writing of the report and decision to submit the paper for publication. 


\section{Conflict of interest}

\section{We have no conflicts of interest to declare}

We also confirm that we had full access to all data in the study, take responsibility for the integrity of the data and the accuracy of the statistical analysis and ensure that each author takes public responsibility for the content.

\section{Acknowledgment}

The authors are grateful for the help of all clinicians and staff at the clinics who took part in this study and wish to thank especially all participants and their families for their collaboration. In addition, we would like to thank K. Daniel O'Leary Distinguished Professor and Director of Clinical Training at the State University of New York who read the manuscript and provided interesting comments on our study.

\section{Contributors}

J. Moya and A. Cano-Prous designed the study and wrote the protocol. J. Moya assessed all participants and elaborated with A. Seva the manuscript. A. Alonso made the statiscal analysis. All authors have approved the final manuscript.

\section{References}

1. Denton WH, Golden, RN, Walsh SR. Depression, marital discord and couple therapy. Curr Opin Psych 2003; 16: 29-34.
2. Weissman M, Paykel ES. The depressed woman: A study of relationships. Chicago: University of Chicago Press; 1974.

3. Beach SR, Arias I, O'Leary KD. The relationship of marital satisfaction and social support to depressive symptomatology. J Psychopathol Behav Assess 1986; 8: 305-316.

4. Dobson KS. Marital and social adjustment in depressed and remitted married women. J Clin Psychology 1987; 43: 261-265.

5. Basco MR, Prager KJ, Pita JM. Communication and intimacy in the marriages of depressed patients. J Fam Psychol 1992; 6: 184-194.

6. Bauserman SA, Arias I. Marital attributions in spouses of depressed patients. J Psychopathol Behav Assess 1995; 17: 231-249.

7. Horneffer KJ, Fincham FD. Construct of attributional style in depression and marital distress. J Fam Psychol 1995; 9:186-195.

8. Frankel KA, Harmon RJ. Depressed mothers: They don't always look as bad as they feel. J Am Acad Child Adolesc Psych 1996; 35: 289-298.

9. Biglan A, Hops H, Sherman L. Problem-solving interactions of depressed women and their husbands. Behav Ther 1985; 16: 431-451.

10. Kurdek LA. The nature and predictors of the trajectory of change in marital quality over the first 4 years of marriage for first-married husbands and wives. J Fam Psychol 1998; 12: 494-510.

11. Whisman M, Bruce ML. Marital distress and incidence of major depressive episode in a community sample. J Abnorm Psychology 1999; 108: 674-678.

12. Davila J, Karney BR, Hall TW, Bradbury TN. Depressive symptoms and marital satisfaction: Within-Subject Association and the Moderating Effects of Gender and Neuroticism. J Fam Psychol 2003; 17: 557-570.

13. Beach SR, Katz J. Prospective effects of marital satisfaction on depressive symptoms in established marriages: A dyadic model. J Soc Pers Relat 2003; 20: 355-371.

14. Whisman M. The Association of Depression and Marital Dissatisfaction. In: Beach SR, ed. Marital and family processes in Depression: A scientific Foundation for Clinical Practice. Washington: American Psychological Association; 2001. p. 3-24.

15. Coyne J, Benazon N. Not Agent Blue: Effects of Marital Functioning on Depression and Implications for Treatment. In: Beach SR, ed. Marital and family processes in Depression: A scientific Foundation for Clinical Practice. Washington, DC: American Psychological Association; 2001. p. 25-45. 
16. Beach SR, O'Leary KD. Marital discord and dysphoria: For whom does the marital relationship predict depressive symptomatology? J Soc Pers Relat 1993; 10: 405-420.

17. Ulrich-Jakubowski D, Russell DW, O'Hara MW. Marital adjustment difficulties: Cause or consequence of depressive symptomatology? J Soc Clin Psychol 1988; 7: 312-318.

18. Keitner GI, Ryan CE, Fodor J, Miller IW. A crosscultural study of family functioning. Contem Fam Ther 1990; 12: 439-454.

19. First MB, Spitzer RL, Gibbon M, Williams JB. Structured Clinical Interview for the DSM-IV Axis I Disorders (SCID-I) research version. Nueva York: New York Psychiatric Institute; 1996.

20. Hamilton M. A rating scale for depression. J Neurol Neuros Psych 1960; 23: 56-62.

21. Endicott J, Spitzer R, Fleiss J, Cohen J. The Global Assessment Scale: a procedure for measuring overall severity of psychiatric disturbance. Arch Gen Psychiatry 1976; 33: 766-771.

22. Williams JB, Gibbon M, First MB, Spitzer RL, Davies M, Borus J, et al. The Structured Clinical Interview for DSM-III-R (SCID). II. Multisite test-retest reliability. Arch Gen Psychiatry 1992; 49(8): 630-636.

23. First MB, Spitzer RL, Gibbon M, Williams JBW. Entrevista clínica estructurada para los trastornos del Eje I del DSM-IV. Versión clínica (SCID-I). Barcelona: Masson, S.A.; 1999.

24. Snaith RP. Present use of the Hamilton Depression Rating Scale: observation on method of assessment in research of depressive disorders. Br J Psychiatry 1996; 168(5): 594-597.

25. Ramos-Brieva JA, Cordero Villafafila A.Validation of the Castillian version of the Hamilton Rating Scale for Depression. Actas Luso Esp Neurol Psiquiatr Cienc Afines 1986; 14(4): 324-34.

26. Startup M, Jackson M, Bendix S. The concurrent validity of the Global Assessment of Functioning (GAF). Br J Clin Psychol 2002; 41(4): 417-422.
27. Spanier GB. Measuring dyadid adjustment: New scales for assessing the quality of marriage. J Mar Fam 1976; 38: 15-28.

28. Crane D, Allgood SM, Larson J. Assessing marital quality with distressed and nondistressed couples. J Mar Fam 1990; 52: 87-93.

29. Spanier GB. Dyadic Adjustment Scale. Association AP. Handbook of psychiatric measures. Washington: American Psychiatric Association; 2000. p. 249-251.

30. Weiss RL, Hops H, Patterson GR. A framework for conceptualizing marital conflict: a technology for offering it, some data for evaluation it. In: Hardy P, Marsh E, eds. Behaviour change: methodology, concepts and practice. Champaign, IL: Research Press; 1973.

31. Weiss R, Margolin G. Assessment of marital conflict and accord. In: Ciminero AR, Calhoun KS, eds. Handbook for Behavioral Assessment. New York: Wiley; 1977. p. 555-602.

32. Margolin, G. Behavioral marital therapy: Is there a place for passion, play, and other non-negotiable dimensions? Behav Ther 1983; 6: 65-58.

33. Baucom D. A comparison of behavioural contracting and problem-solving/communication training in behavioural marital therapy. Behav Ther 1982; 13: 162-174.

34. Stravynski A, Tremblay M, Verreault R. Marital adjustment and depression. Psycholopathology 1995; 28: 112-117.

35. Vega BR, Cañas F, Bayón C. Interpersonal factors in female depression. Eur J Psych 1996; 10: 16-24.

36. Hooley JM, Teasdale JD. Predictors of relapse in unipolar depressives: Expressed emotion, marital distress, and percieved criticism. J Abnorm Psychology 1989; 98: 229-235.

Address for correspondence:

Hospital Clínic Barcelona

C/ Villarroel, 170

08036 Barcelona, Spain

Tel: 9322754 00/ 932279974

Fax: 932279172

E-mail: jmoya1@clinic.ub.es 